\title{
Formation of Metal Nanoparticles in Multilayered Poly(octadecylsiloxane) As Revealed by Anomalous Small-Angle X-ray Scattering
}

\author{
Dmitri I. Svergun, ${ }^{*, t, \neq}$ Mikhail B. Kozin,,$+\neq$ Peter V. Konarev, ${ }^{\dagger, \neq}$ \\ Eleonora V. Shtykova, ${ }^{\dagger}$ Vladimir V. Volkov, ${ }^{\dagger}$ Dmitri M. Chernyshov, ${ }^{\S}$ \\ Peter M. Valetsky, ${ }^{\S}$ and Lyudmila M. Bronstein $n^{\S, \perp}$ \\ Institute of Crystallography, Russian Academy of Sciences, Leninsky pr. 59, 117333 Moscow, \\ Russia, EMBL, Hamburg Outstation, Notkestrasse 85, D-22603 Hamburg, Germany, \\ Nesmeyanov Institute of Organoel ement Compounds, Russian Academy of Sciences, \\ Vavilov St. 28, Moscow 117813, Russia, and Chemistry Department, Indiana University, \\ Bloomington, Indiana 47405
}

Received J une 16, 2000. Revised Manuscript Received August 14, 2000

\begin{abstract}
Novel hybrid polymeric systems with noble metal nanoparticles located inside specific areas of the material were developed. A self-assembled multilayered polymer, poly(octadecylsiloxane) (PODS), provided a nanostructured matrix for incorporation of gold and platinum compounds and for metal nanoparticle formation. The internal structure of PODS and the nanoparticle size distributions were examined using anomal ous small-angle X-ray scattering. The ordering in PODS was largely preserved after interaction with metal compounds and reducing agents. The degree of incorporation of the compounds into PODS depended on the reaction conditions and on the compound type. For the metal-containing PODS isolated from the reaction medium before reduction, the major fraction of the nanoparticles had radii around $2 \mathrm{~nm}$, and the size distributions depended neither on the compound loading nor on the reducing agent. This points to a "cage"-controlled particle growth restricted by the cavity size in the siloxy bilayer. This hypothesis is corroborated by the computed density profiles across the PODS Iamella. I ncor poration of cetyl pyridinium chloride in the hydrophobic layers of PODS promotes formation of nanoparticles also between the hydrophobic tails. This location does not restrict the particle growth so that the nanoparticle sizes strongly depend on the reduction conditions.
\end{abstract}

\section{Introduction}

Nanosized metal particles have huge surface areas and possess unique properties that can be employed in a wide range of chemical and physical applications: catalysis, nonlinear optics, semiconductors, microelectronics, and others. ${ }^{1-3}$ Properties of metal colloids depend strongly on the particle shapes, size distributions, and chemical environment. Use of a polymeric matrix as a medium for metal nanoparticle formation stabilizes particles and controls the nanoparticle growth, yet imparting new properties to the matrix itself. A better control of the nanoparticle growth is achieved in nanostructured matrices such as amphiphilic block copolymers in bulk, block copolymer micelles, etc. ${ }^{4-9}$

I Institute of Crystallography, Russian Academy of Sciences.

₹EMBL, Hamburg Outstation.

$\S$ Nesmeyanov Institute of Organoelement Compounds, Russian Academy of Sciences.

$\perp$ Indiana University.

* Corresponding author: Tel +49 4089902 125, Fax +49 4089902

149, E-mail Svergun@EMBL-Hamburg.DE.

(1) Henglein, A. Chem. Rev. 1989, 89, 1861.

(2) Oggawa, S.; Hayashi, Y.; Kobayashi, N.; Tokizaki, T.; Nakamura, A. J pn. J . Appl. Phys. 1994, 33, L331.

(3) Nanoparticles and Nanostructured Films; Fendler, J . H., Ed.; Wiley-VCH: New York, 1998.

(4) Bradley, J. The Chemistry of Transition Metal Colloids. In Clusters and Colloids; Schmid, G., Ed.; Wiley-VCH: New York, 1994.
Most well-defined amphiphilic block copolymers (most efficient in nanoparticle control) are still synthesized by anionic polymerization, thereby demanding a high degree of purification at all the steps of synthetic procedure. A very robust alternative way for preparation of metal nanoparticles in an ordered polymeric environment in aqueous media is proposed in the present paper.

A procedure to synthesize highly ordered layered polymers via hydrolytic polycondensation of n-octadecyltrichlorosilane (PODS) in water has recently been proposed. ${ }^{10-12}$ It has been demonstrated ${ }^{12}$ that the polymeric condensate consists of highly uniform, pillared microcrystallites in which the inorganic siloxy backbones form periodic layers, each containing a monomolecular layer of intercalated water, separated by crystalline assemblies of alkyl chains. Being placed

(5) Antonietti, M.; Wenz, E.; Bronstein, L.; Seregina, M. Adv. Mater. 1995, 7, 1000.

(6) Chan, Y. N. C.; Schrock, R. R.; Cohen, R. E. Chem. Mater. 1992, $4,24$.

(7) Saito, H.; Okamura, S.; Ishizu, K. Polymer 1992, 33, 1099.

(8) Moffit, M.; McMahon, L.; Pessel, V.; Eisenberg, A. Chem. Mater. 1995, 7, 1185.

(9) Spatz, J . P.; Roescher, A.; Möller, M. Adv. Mater. 1996, 8, 337.

(10) Thompson, W. R.; Pemberton, J. E. Langmuir 1995, 11, 1720.

(11) Gao, W.; Reven, L. Langmuir 1995, 11, 1860.

(12) Parikh, A. N.; Schivley, M. A.; Koo, E.; Seshadri, K.; Aurentz, D.; Mueller, K.; Allara, D. L. J . Am. Chem. Soc. 1997, 119, 3135. 
Table 1. Reaction Conditions and Metal Content in the Samples

\begin{tabular}{|c|c|c|c|c|c|}
\hline \multirow[b]{3}{*}{ sample notation } & \multirow[b]{3}{*}{ reaction medium } & \multirow[b]{3}{*}{ reducing agent and medium } & \multicolumn{3}{|c|}{ metal content, wt \% } \\
\hline & & & \multirow[b]{2}{*}{ totald } & \multicolumn{2}{|c|}{ in nanoparticles e } \\
\hline & & & & total & small/large \\
\hline PODS1-Pt(1)a & water/acetone & self-reduction & 1.20 & 0.22 & $86 / 14$ \\
\hline PODS1-Pt(2) ${ }^{b}$ & water/ethanol & $\mathrm{H}_{2}$, mother solution & 0.80 & $-f$ & \\
\hline PODS1-Pt(3) & water/ethanol & $\mathrm{NaBH}_{4}$, mother solution & 0.76 & $-f$ & \\
\hline PODS1-Pt(4) & water/acetone & $\mathrm{H}_{2}$, water/acetone & 0.56 & 0.35 & $88 / 12$ \\
\hline PODS1-Pt(5) & water/acetone & $\mathrm{NaBH}_{4}$, water/acetone & 0.48 & 0.36 & $81 / 19$ \\
\hline PODS1-Au(1)c & water/acetone & self-reduction & 0.60 & 0.17 & $23 / 77$ \\
\hline PODS1-Au(2) & water/ethanol & self-reduction & 0.80 & 0.14 & 19/81 \\
\hline PODS2/CPC-Au(3) & water & $\mathrm{NaBH}_{4}$, water & 1.92 & 0.75 & $69 / 31$ \\
\hline PODS2/CPC-Au(4) & water & UV irradiation, solid state & 1.66 & 0.29 & $26 / 74$ \\
\hline
\end{tabular}

${ }^{a} \mathrm{~K}_{2} \mathrm{PtCl}_{4}$ was used for preparation of PODS-Pt1. ${ }^{b} \mathrm{H}_{2} \mathrm{PtCl}_{6} \cdot 6 \mathrm{H}_{2} \mathrm{O}$ was used for synthesis of the PODS-Pt(2-5) samples. ${ }^{\mathrm{c}} \mathrm{F}$ or synthesis of the PODS-Au samples, $\mathrm{AuCl}_{3}$ was employed. d By elemental analysis. e Computed from the size distributions. ${ }^{\mathrm{f}} \mathrm{N}$ ot computed. $9 \mathrm{Relative}$ volume of particles with radii less/more than $5 \mathrm{~nm}$ in percent.

in an aqueous solution of metal salt, this polymer system is expected to absorb salt by its hydrophilic layer. The subsequent reduction of the metal compound would yield a novel polymeric material containing regularly spaced metal nanoparticles.

We employed PODS as a matrix for the metal nanoparticle formation and characterized the structure of the obtained systems by small-angle X-ray scattering (SAXS). The latter diffraction method is widely used to analyze the structure of various disperse systems at a resolution from about 1 to $100 \mathrm{~nm}$. This method has recently been successfully employed to study the internal structure and metal nanoparticle formation in polyelectrolyte gel/ surfactant complexes. ${ }^{13-16}$ When studying metal particles in a polymer environment, anomalous scattering (ASAXS) is of special interest. Recording scattering patterns at beam energies near the absorption edge of the metal enables an in situ separation of the scattering contributions from metal nanoparticles and the polymer martix. ${ }^{17,18}$ Analysis of these contributions permits to compute the metal particle size distributions and to investigate the internal structure of the matrix. In the present paper we report an ASAXS analysis of the internal PODS structure and of the metal nanoparticle formation in PODS for different reducing agents and reaction media. The size distributions of the noble metal nanoparticles are computed, and changes in the internal structure of the ordered polymer caused by the metal nanoparticle growth are monitored.

\section{Experimental Section}

Materials and Sample Preparation. Reagent grade quality acetone and ethanol (Aldrich) were used after distillation. $\mathrm{NaBH}_{4}$ (Reidel-de-Haen), cetylpyridinium chloride (CPC), $\mathrm{K}_{2} \mathrm{PtCl}_{4}, \mathrm{AuCl}_{3}$, and $\mathrm{H}_{2} \mathrm{PtCl}_{6} \cdot 6 \mathrm{H}_{2} \mathrm{O}$ (Aldrich) were used

(13) Dembo, A. T.; Yakunin, A. N.; Zaitsev, V. S.; Mironov, A. V.; Starodubtsev, S. G.; Khokhlov, A. A.; Chu, B. J . Polym. Sci., Polym. Phys. 1996, 34, 2893.

(14) Bronstein, L. M.; Platonova, O. A.; Yakunin, A. N.; Yanovskaya, I. M.; Valetsky, P. M.; Dembo, A. T.; Makhaeva, E. E.; Mironov, A. V.; Khokhlov, A. R. Langmuir 1998, 14, 252

(15) Bronstein, L. M.; Platonova, O. A.; Yakunin, A. N.; Yanovskaya, I. M.; Valetsky, P. M.; Dembo, A. T.; Obolonkova, E. S.; Makhaeva, E E.; Mironov, A. V.; Khokhlov, A. R. Colloids Surf. 1999, A147, 221.

(16) Svergun, D. I.; Shtykova, E. V.; Dembo, A. T.; Bronstein, L. M.; Platonova, O. A.; Yakunin, A. N.; Valetsky, P. M.; Khokhlov, A. R. J. Chem. Phys. 1998, 109, 11109.

(17) Stuhrmann, H. B.; Goerigk, G.; Munk, B. Anomalous X-ray Scattering. In Handbook on Synchrotron Radiation; Rubenstein, E., Ebashi, S., Koch, M., Eds.; Elsevier: Amsterdam, 1994; p 555.

(18) Haubold, H.-G.; Wang, X. H.; Goerigk, G.; Schilling, W. J . Appl . Crystallogr. 1997, 30, 653. as received. Water was purified with a Milli-Q water purification system.

The poly(octadecylsiloxane) samples were synthesized by the method described in ref 12 . The metal-containing polymers were prepared by stirring the PODS powder $(0.1 \mathrm{~g})$ in $10 \mathrm{~mL}$ of water/acetone (1/1) or of water/ethanol (1/1) solution of the appropriate metal salt for $24 \mathrm{~h}$. The concentration of the metal salt in solution was $10^{-3} \mathrm{~mol} / \mathrm{L}$. The metal-containing pol ymer gels were isolated by filtration and thoroughly washed from unreacted metal salt with water/acetone (1/1) mixture. The collected powder was then dried in the vacuum desiccator overnight. Elemental analysis gave the following composition, \%: C, 69.9; $\mathrm{H}, 11.2$; $\mathrm{Si}, 8.35 ; \mathrm{O}, 10.1$. For the formula $\mathrm{C}_{18} \mathrm{H}_{39-}$ $\mathrm{Si}_{1.0} \mathrm{O}_{2.5}$ (fully cross-linked octadecylsiloxane moieties with no water incorporation), calculated values are as follows,\%: C, 70.80; H, 12.13; Si, 9.18; O, 7.87.

The PODS/CPC complexes were prepared using absorption of surfactant molecules by PODS powder in water. For this, dry PODS $(0.1 \mathrm{~g})$ was added to the $0.005 \mathrm{~mol} / \mathrm{L} \mathrm{CPC}$ solution (critical micelle concentration for CPC is $0.0014 \mathrm{~mol} / \mathrm{L}$ ) and stirred at room temperature for $12 \mathrm{~h}$. After addition of PODS powder, the initially turbid surfactant solution turned transparent within $1-2 \mathrm{~h}$. The resulting PODS/CPC suspension was filtered and dried in a vacuum desiccator overnight. Elemental analysis gave the following composition, \%: C, 70.8; $\mathrm{H}, 11.8$; $\mathrm{Si}, 6.45 ; \mathrm{N}, 0.74 ; \mathrm{Cl}, 1.92 ; \mathrm{O}, 8.03$. The metal-containing PODS suspensions were reduced with $\mathrm{NaBH}_{4}$ or $\mathrm{H}_{2}$ either in mother solution (containing the corresponding metal salt) or after isolation of metalated PODS sample and its redispersion in the medium (see Table 1 for details). Sodium borohydride reduction of the metal-containing PODS suspensions was performed either in water/acetone or in water/ethanol media. For this, the reaction solutions were charged with an appropriate amount of $\mathrm{NaBH}_{4}$ (5-fold molar excess with respect to metal content) and stirred for $4 \mathrm{~h}$. After addition of the reducing agent a rapid darkening of the metalated PODS powder was observed. In the $\mathrm{H}_{2}$ reduction, typically, molecular hydrogen was bubbled through the reaction mixture at room temperature for approximately $15 \mathrm{~min}$ followed by stirring of the suspension for additional $2 \mathrm{~h}$ in the sealed reaction vessel. The resulting product was isolated, thoroughly washed with a water/ethanol or water/acetone, and dried in a vacuum desiccator for $12 \mathrm{~h}$. For a radiation-induced reduction, a dried highly dispersed PODS powder was carefully placed onto a surface of a quartz dish, dispersed in a thin layer, and UV irradiated for $3 \mathrm{~h}$.

Scattering Experiments and Data Analysis. The ASAXS measurements were performed at the beamline J USIFA ${ }^{19}$ on the storage ring DORIS III of the Deutsches Elektronen Synchrotron (DESY, Hamburg). Anomalous scattering experiments involve measurements of the scattering curves at different X-ray radiation wavelengths $\lambda$, i.e., for different

(19) Haubold, H.-G.; Gruenhagen, K.; Wagener, M.; J ungbluth, H.; Heer, H.; Pfeil, A.; Rongen, H.; Brandenburg, G.; Moeller, R.; Matzerath, J.; Hiller, P.; Halling, H. Rev. Sci. Instrum. 1989, 60, 1943. 
energies of the incident beam E.17,20,21 The atomic scattering factor is

$$
f(E)=f_{0}+f^{\prime}(E)+\text { if }{ }^{\prime \prime}(E)
$$

where the anomalous dispersion corrections $f^{\prime}(E)$ and $f^{\prime \prime}(E)$ to $f(E)$ become significant if the energy of the incident beam is close to that of an X-ray absorption edge of the atom. The samples were measured near the $L_{3}$ absorption edges of $A u$ and $\mathrm{Pt}$ at the photon energies around $\mathrm{E}=11.9$ and $11.5 \mathrm{keV}$ $(\lambda=0.104$ and $0.107 \mathrm{~nm}$, respectively). Two sample-detector distances, 935 and $3635 \mathrm{~mm}$, covered the ranges of the momentum transfer $0.4<\mathrm{s}<7.3 \mathrm{~nm}^{-1}$ and $0.08<\mathrm{s}<1.5$ $\mathrm{nm}^{-1}$, respectively ( $\mathrm{s}=4 \pi \sin \theta / \lambda$, where $2 \theta$ is the scattering angle). The sample chamber was evacuated to reduce the parasitic scattering, and the PODS powders were measured in specially designed $1 \mathrm{~mm}$ thick aluminum cuvettes with mica windows. The scattering profiles at the two sample-detector distances were corrected for the background scattering and fluorescence, cal ibrated into absol ute units following standard procedures, and merged to yield the composite curves in the range $0.08<\mathrm{s}<7.3 \mathrm{~nm}^{-1}$.

For each sample, the scattering curves $I\left(s, E_{k}\right)$ were recorded at four energies of the incoming photons $k=0, \ldots, 3$ in the vicinity of the absorption edge of the corresponding metal. The first energy was selected to be sufficiently far from the edge $\left(E_{0}=11.560\right.$ and $11.487 \mathrm{keV}$ for $\mathrm{Au}$ and Pt samples, respectively). For the three energies close to the edge $\left(E_{1,2,3}=11.870\right.$, 11.915 , and $11.919 \mathrm{keV}$ for $\mathrm{Au}$ and $11.535,11.555$, and 11.564 $\mathrm{keV}$ for $\mathrm{Pt}$ ), the anomalous corrections amounted to approximately $-0.06 f\left(E_{0}\right),-0.10 f\left(E_{0}\right)$, and $-0.20 f\left(E_{0}\right)$, respectively. The differences between the curves recorded at different energies $\Delta_{k}(\mathrm{~s})=\mathrm{I}\left(\mathrm{s}, \mathrm{E}_{0}\right)-\mathrm{I}\left(\mathrm{s}, \mathrm{E}_{\mathrm{k}}\right)$, proportional to the scattering from the metal atoms, were used to compute the size distribution functions. Assuming the particles to be spherical, an indirect transform program $\mathrm{GNOM}^{22}$ was employed to solve the integral equation

$$
\mathrm{I}(\mathrm{s})=(\Delta \rho)^{2} \int_{\mathrm{R}_{\min }}^{\mathrm{R}_{\max }} \mathrm{D}_{\mathrm{V}}(\mathrm{R}) \mathrm{m}^{2}(\mathrm{R}) \mathrm{i}_{0}(\mathrm{sR}) \mathrm{dR}
$$

where $R$ is the radius of a sphere, $R_{\min }$ and $R_{\max }$ are the minimum and maximum radii, respectively, and $\mathrm{i}_{0}(\mathrm{x})=$ $\left\{[\sin (x)-x \cos (x)] / x^{3}\right\}^{2}$ and $m(R)=(4 \pi / 3) R^{3}$ are the sphere form factor and volume, respectively. The scattering length density of the anomalous atoms is $\Delta \rho=\left(\mathrm{N}_{0}{ }^{2}-\mathrm{N}_{\mathrm{k}}{ }^{2}\right) \mathrm{e} / \mathrm{v}_{\mathrm{at}}$ where $\mathrm{N}$ and $\mathrm{N}_{\mathrm{k}}$ are numbers of electrons contributing to the scattering far from the resonance and at $\lambda=\lambda_{\mathrm{k}}$, e is the Thompson radius of an el ectron, and $v_{a t}$ is the atomic volume. $\mathrm{F}$ or $\mathrm{k}=3$ (maximum anomalous signal) $\Delta \rho=6.80 \times 10^{11}$ and $6.94 \times 10^{11} \mathrm{~cm}^{-2}$ for $\mathrm{Pt}$ and $\mathrm{Au}$, respectively. In the computations of $D_{v}(R)$, the value of $R_{\min }$ was kept zero; that of $R_{\max }$ was selected for each individual data set by successive runs with different values of this parameter.

The characteristics of the internal structure of the polymer matrix were obtained by fitting Gaussian profiles to interactively selected Bragg peaks in the scattering patterns using the program PEAK (Konarev and Svergun, unpublished). The long-range order dimension $L$ and the degree of disorder in the system $\Delta / \overline{\mathrm{d}}$ were calculated as described elsewhere. ${ }^{23}$

$$
\begin{gathered}
\mathrm{L}=\frac{\lambda}{\beta_{\mathrm{s}} \cos \theta} \\
\Delta / \overline{\mathrm{d}}=\frac{1}{\pi} \sqrt{\frac{\beta_{\mathrm{s}} \overline{\mathrm{d}}}{\lambda}}
\end{gathered}
$$

where $\beta_{\mathrm{s}}$ is the full width at a half-maximum intensity of the peak (in radians) observed at a mean scattering angle of $2 \theta, \bar{d}$

(20) Haubold, H.-G.; Gebhardt, R.; Buth, G.; Goerigk, G. In Resonant Anomal ous X-ray Scattering; Materlik, G., Sparkks, C. J . Fischer, K., Eds.; Elsevier Science: Oxford, 1994; pp 295-304.

(21) Haubold, H.-G. J . Phys. IV C8, (Suppl. J . Phys. I) 1993, 3, 475.
$=2 \pi / s_{\max }$ is the characteristic size of the crystalline regions corresponding to the peak position $S_{\max }$, and $\Delta$ is the meansquare deviation of distances between the neighboring layers.

The centrosymmetric density profiles along the direction perpendicular to the lamella plane of the multilayers were computed by a one-dimensional Fourier transformation

$$
\rho(r)=\sum_{I=1}^{N} s_{l} A(s, I)=\sum_{I=1}^{N} s_{l} \sin \pi s r[ \pm \sqrt{S(I)}]
$$

where $\mathrm{N}$ is the number of peaks and $\mathrm{s}$ and $\mathrm{S}(\mathrm{I})$ are the position and the area of the Ith Bragg peak, respectively. All combinations of signs of $A(s, I)$ were considered, and the most plausible was selected by a visual inspection of the density profiles. The latter were low pass filtered over the period of the termination wave $T=2 \pi / S_{N}$ to reduce the termination effect.

\section{Results and Discussion}

1. Synthesis of Metal-Containing PODS. Several procedures were employed for incorporation of metal compounds of different types. Table 1 presents the conditions of the metal compound incorporation and the metal loading values in the hybrid materials obtained by elemental analysis data. Water/acetone (1/1) and water/ethanol (1/1) served as reaction media. The necessity of an organic solvent presence in the medium was dictated by high hydrophobicity of the PODS exterior, while the hydrophilic layers were located inside the material. ${ }^{12}$

According to ref 12 , the interior siloxy bilayer contains $(-\mathrm{SiO})_{x}(\mathrm{OH})_{y}$ sheets with relative populations of about 25 and $75 \%$ for $\mathrm{Si}$ atoms with $\mathrm{y}=0$ and 1 , respectively. This indicates that a significant portion of silanol groups can interact with the monolayer of water through hydrogen bonds. ${ }^{12} \mathrm{As}$ known, the platinum ions ( $\mathrm{PtCl}_{6}{ }^{2-}$ or $\mathrm{PtCl}_{4}{ }^{2-}$ ) are capable of forming complexes with $\mathrm{NH}^{+}$ groups through hydrogen bonding. ${ }^{24} \mathrm{E}$ arlier, we studied the interaction of $\mathrm{NH}$ groups of polyethylenimine with $\mathrm{NaPtCl}_{6}$, which resulted in micellization due to the hydrogen bond formation.25,26 We expected that the silanol groups are also able to interact with the above Pt ions due to hydrogen bonding, and this might be a driving force for the ion incorporation into the siloxy bilayer. The main questions were whether this driving force is sufficiently strong to compete with the already existent hydrogen bonds in the bilayers and which kind of metal ions is more competitive in this process.

For comparison, we studied the incorporation of octahedral $\mathrm{PtCl}_{6}{ }^{2-}$ (from $\mathrm{H}_{2} \mathrm{PtCl}_{6} \cdot 6 \mathrm{H}_{2} \mathrm{O}$ ) and of planar $\mathrm{PtCl}_{4}{ }^{2-}$ (from $\mathrm{K}_{2} \mathrm{PtCl}_{4}$ ). The incorporation of $\mathrm{H}_{2} \mathrm{PtCl}_{6} \cdot$ $6 \mathrm{H}_{2} \mathrm{O}$ followed by reduction was carried out in two ways as described in the Experimental Section. If the reduction is performed in mother solution, the resulting Ptcontaining PODS may carry large particles precipitated from the solution onto the PODS surface. To avoid this, the hybrid material was isolated after $24 \mathrm{~h}$ stirring in the presence of $\mathrm{H}_{2} \mathrm{PtCl}_{6} \cdot 6 \mathrm{H}_{2} \mathrm{O}$ and then dispersed in acetone/water medium and reduced (Table 1 ). This

(22) Svergun, D. I. J . Appl. Crystallogr. 1992, 25, 495.

(23) Vainshtein, B. K. Diffraction of X-rays by Chain Molecules; Elsevier Publishing Company: Amsterdam, 1966.

(24) Valle, G.; Ettorre, R. Acta Crystallogr. 1998, C54, 448.

(25) Bronstein, L.; Sidorov, S.; Gourkova, A.; Valetsky, P.; Hartmann, J .; Bruelmann, M.; Cölfen, H.; Antonietti, M. Inorg. Chim. Acta. 1998, 280, 348.

(26) Sidorov, S.; Bronstein, L.; Valetsky, P.; Hartmann, J .; Cölfen, H.; Schnablegger, H.; Antonietti, M. J . Colloi d I nterfaceSci. 1999, 212, 197. 
(c)

(a)

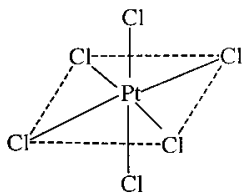

(b)

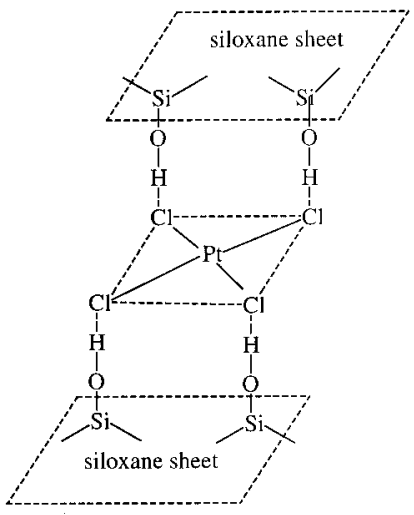

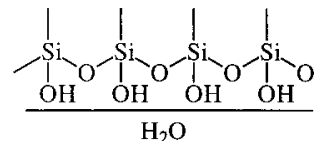

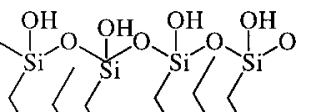
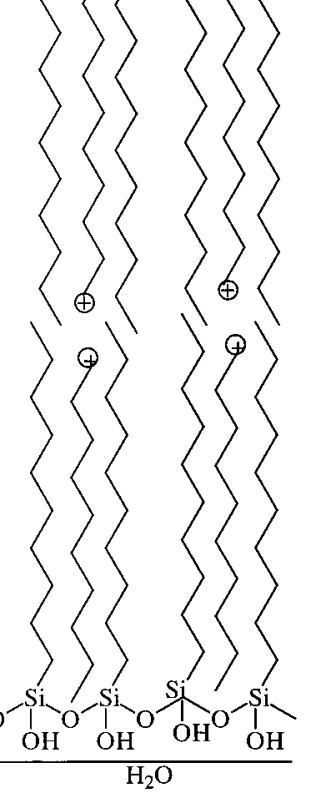

O $\mathrm{SH}_{\mathrm{Si}}^{\mathrm{OH}} \mathrm{OH}_{\mathrm{Si}}^{\mathrm{OH}} \mathrm{OH}_{\mathrm{Si}}^{\mathrm{OH}}-\mathrm{O}_{\mathrm{Si}}^{\mathrm{OH}}$

Figure 1. (a) Geometry of the $\mathrm{PtCl}_{6}{ }^{2-}$ ion. (b) Schematic interaction of the planar $\mathrm{K}_{2} \mathrm{PtCl}_{4}$ ion with silanol groups of the siloxy bilayer. (c) Schematic image of PODS/CPC hybrid (hydrocarbon tails are shown shorter than they are).

permitted to avoid the precipitation of the large particles, but the Pt loading in the resulting material was not high. This indicates that penetration of the $\mathrm{PtCl}_{6}{ }^{2-}$ ions in the siloxy bilayer (which is the only way PODS may acquire polar metal ions) is not very intense.

We assumed that the low degree of penetration might be caused by geometry of the $\mathrm{PtCl}_{6}{ }^{2-}$ ion presented in Figure 1a. Penetration of this ion into the siloxy bilayer might disturb the latter while the advantages of hydrogen bond formation of silanol groups with $\mathrm{PtCl}_{6}{ }^{2-}$ compared to water molecules are questionable: not more than two water molecules can be replaced by one anion. An alternative Pt compound is a planar $\mathrm{K}_{2} \mathrm{PtCl}_{4}$, for which the interaction with silanol groups of the siloxy bilayer can be envisioned as in Figure 1b. Here, $\mathrm{Pt}$ ion incorporation should not disturb the sheet structure of the siloxane bilayers, and formation of four hydrogen bonds with each Pt ion can replace up to four water molecules. This increases the entropy, and the latter is thus an important driving force for ion penetration.

The incorporation of $\mathrm{K}_{2} \mathrm{PtCl}_{4}$ in PODS was performed in the way similar to $\mathrm{H}_{2} \mathrm{PtCl}_{6} \cdot 6 \mathrm{H}_{2} \mathrm{O}$, but al ready in $4 \mathrm{~h}$ the color of the PODS powder turned from light pink to dark brown, indicating the compound reduction. In the absence of PODS, $\mathrm{K}_{2} \mathrm{PtCl}_{4}$ remained unchanged in the water/acetone medium. This means that reduction of the $\mathrm{PtCl}_{4}{ }^{2-}$ ions is possible only due to silanol groups of PODS; thus, all the Pt ions should be incorporated in the siloxy bilayer where the reduction occurs. The elemental analysis yielded a higher degree of Pt incorporation in PODS compared to $\mathrm{H}_{2} \mathrm{PtCl}_{6}$ (Table 1).

The different behavior of $\mathrm{Pt}$ ions toward a mild reducing agent (silanol groups) can be correlated with their ability to be reduced in aqueous solutions, which can be approximately estimated by standard (or formal) potentials. According to ref 27, standard reduction potentials of $\mathrm{PtCl}_{6}{ }^{2-}$ and $\mathrm{PtCl}_{4}{ }^{2-}$ ions to metal atoms are +0.557 and $+0.749 \mathrm{eV}$, respectively. Though these values were obtained in different conditions, ${ }^{27}$ they demonstrate the same tendency, namely, an easier reduction of $\mathrm{PtCl}_{4}{ }^{2-}$ as compared to $\mathrm{PtCl}_{6}{ }^{2-}$.

The functionality of PODS due to silanol groups in thin siloxy bilayers provides a sufficient driving force for active incorporation of particular metal ions $\left(\mathrm{PtCl}_{4}{ }^{2-}\right)$ inside the layered polymer. To further enhance the opportunities for incorporation of metal compounds, a complex of PODS with a cationic surfactant, CPC, was prepared. The surfactant molecules are expected to enter PODS due to hydrophobic interactions. An exchange of surfactant counterions in such a hybrid system for metal anions would provide an active metal compound incorporation as observed for hybrid micelles of amphiphilic diblock copolymers with surfactants. ${ }^{28}$ As the presence of surfactant increases the hydrophobicity of the reaction medium, the inclusion of CPC into PODS could be carried out in water (without any organic solvent). At the same time, hydrophilicity of PODS was increased due to CPC charged groups, canceling the repulsion between PODS and water molecules. By elemental analysis (see Experimental Section), initial PODS matches stoichiometry of $\mathrm{C}_{18} \mathrm{H}_{37} \mathrm{Si}_{0.92} \mathrm{O}_{1.95}$, close to the one described in ref 12 . The interaction with CPC results in the formation of a hybrid unit $\left(\mathrm{C}_{18} \mathrm{H}_{37^{-}}\right.$ $\left.\mathrm{Si}_{0.92} \mathrm{O}_{1.95}\right)_{4.7} \cdot \mathrm{C}_{21} \mathrm{H}_{38} \mathrm{NCl}$ containing nearly one CPC molecule per 5 siloxy units.

The resulting hybrid PODS/CPC system was dispersed in water and was subjected to the interaction with a metal compound for $24 \mathrm{~h}$. The $\mathrm{AuCl}_{3}$ salt was used as a metal source because after dissolution in water it forms a monovalent planar $\mathrm{AuCl}_{3}(\mathrm{OH})^{-}$ion whose incorporation should not disturb the $\mathrm{CPC}$ packing (unlike bivalent ions $\mathrm{PtCl}_{6}{ }^{2-}$ and $\mathrm{PtCl}_{4}{ }^{2-}$ ). Table 1 presents the loading values for the PODS/CPC-Au system. For comparison, two Au-containing samples were also prepared in PODS without CPC. For these two samples, the gold salt was reduced by the reaction medium (water/ethanol or water/acetone) al ready within 15 min after addition, and therefore penetration of the gold ions inside PODS could hardly be expected.

The incorporation of CPC molecules in PODS can occur in two ways. First, the polar CPC headgroups can be located in vicinity of the polar siloxy bilayer. To form this structure, very hydrophilic pyridinium groups should move within a very hydrophobic environment (octadecyl chains). The probability of this event is extremely low. Second, the hydrophobic tails of the surfactants can move between the hydrophobic tails of PODS so that the incorporation is driven by hydrophobic interactions. As a turbid micellar solution becomes clear in the presence of PODS, one can conclude that the

(27) Encycl opedia of Electrochemistry of the Elements; Bard, A. J ., Ed.; M. Dekker: New York, 1973.

(28) Bronstein, L.; Chernyshov, D.; Valetsky, P.; Timofeeva, G.; Dubrovina, L.; Khokhlov, A.; Obolonkova, E. Langmuir 2000, 16, 3626. 
$\lg$ I, relative

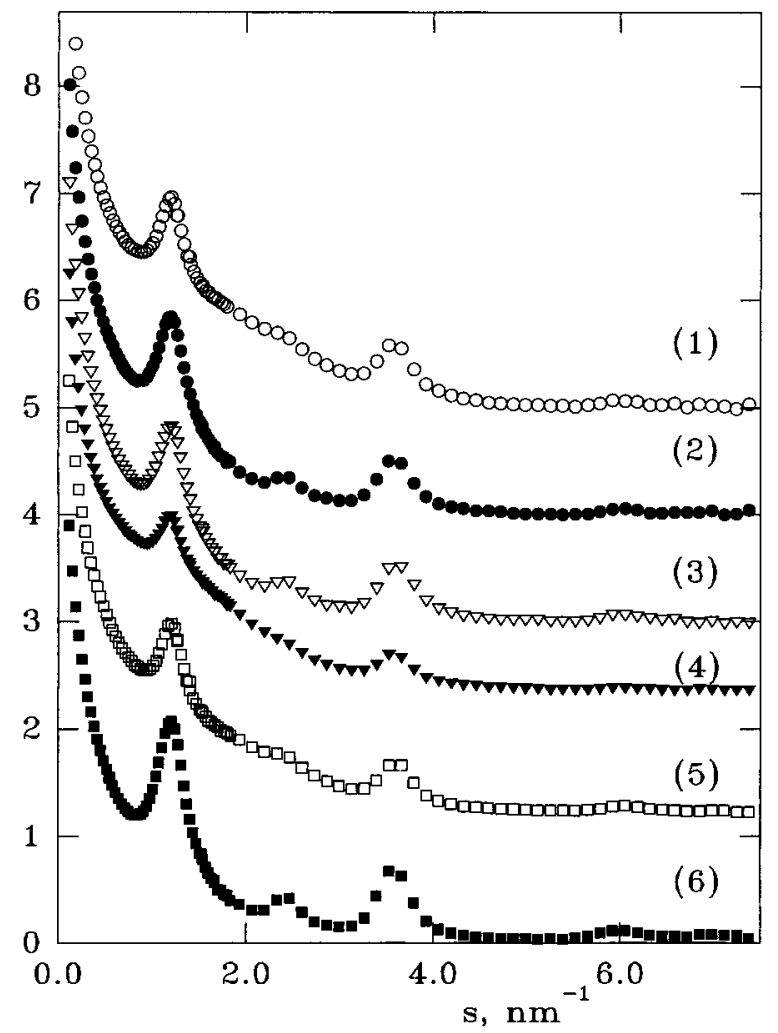

Figure 2. SAXS patterns from the Pt-containing PODS powders far from the absorption edge. Curves 1-5 correspond to the samples Pt1, Pt2, Pt3, Pt4, and Pt5, respectively. The successive curves are displaced one logarithmic unit down for better visualization. The statistical error bars in the data are smaller than the symbol size.

latter interactions are even more favorable for the CPC molecules then those of the CPC micelle formation. As discussed above, PODS has a very hydrophobic exterior, repelling water molecules, and cannot even be dispersed in water. ${ }^{12}$ After incorporation of CPC, PODS becomes sufficiently hydrophilic and can be easily dispersed in water under mild heating $\left(40-50{ }^{\circ} \mathrm{C}\right)$. On the basis of these facts, the incorporation of CPC in PODS can be envisioned as in Figure 1c. According to this scheme, the interaction of metal anions, for example, $\mathrm{AuCl}_{3}(\mathrm{OH})^{-}$, with CPC headgroups due to el ectrostatic forces should mainly occur in the hydrophobic bilayer. On the other hand, one should keep in mind that the planar ions $\mathrm{AuCl}_{3}(\mathrm{OH})^{-}$can penetrate the hydrophilic siloxy bilayer as well. F or the PODS/CPC system, the metal reduction was carried out in two ways: chemically (with $\mathrm{NaBH}_{4}$ ) and photochemically (by UV irradiation). The latter way was examined to check the assumption that the gold ions are located in the hydrophobic layers of PODS where they may be exposed to UV irradiation.

2. ASAXS Study of Metal-Containing PODS. 2.1. Platinum-Containing PODS. 2.1.1. Size Distributions. The experimental scattering profiles from the Ptcontaining PODS measured at beam energy far from the absorption edge $\left(E=E_{0}\right)$ are presented in Figure 2 (curves 1-5) al ong with the scattering from the initial PODS powder (curve 6). To obtain the scattering from the Pt particles, differences between the SAXS intensities at different energies were calculated as described above. Two criteria were used to verify that the difference signal was indeed due to the metal particles: (i) $\lg \mathrm{I}, \mathrm{cm}^{2} / \mathrm{cm}^{3}$

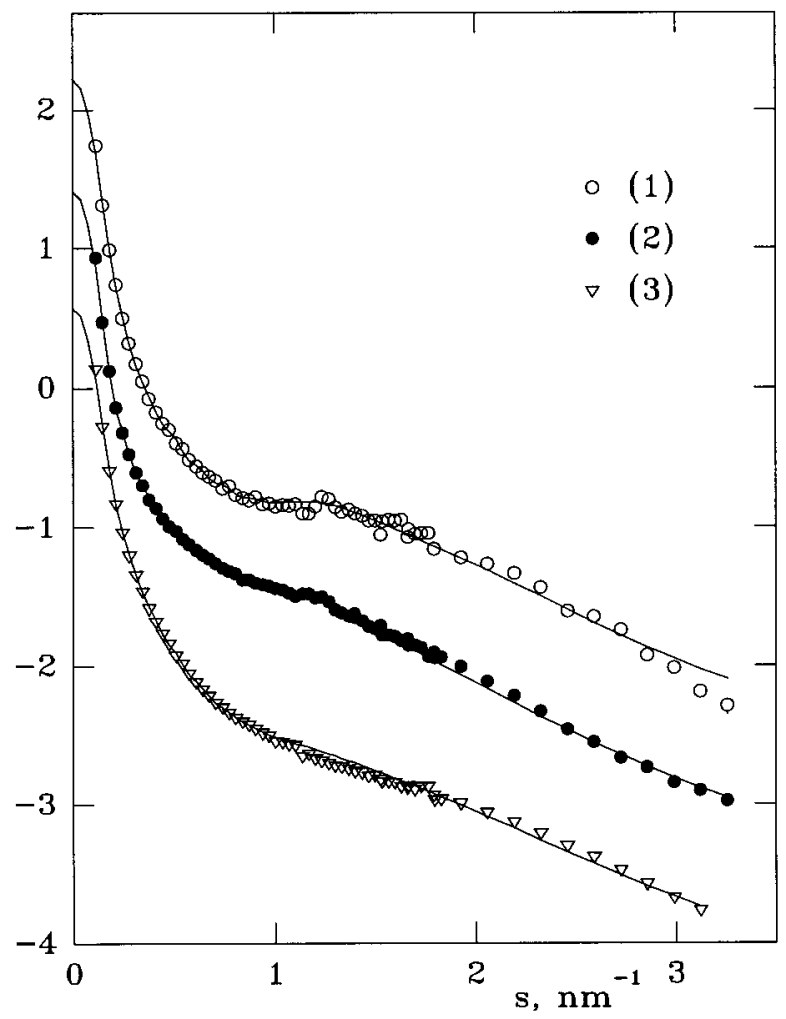

Figure 3. Difference ASAXS data (anomalous signal) from the Pt-containing PODS samples. The data sets are numbered as in Figure 2. Symbols with error bars: experimental data. Solid lines: back-transformed curves from the volume distribution functions computed by GNOM. The successive curves are displaced one logarithmic unit down for better visualization.

the difference $\Delta_{k}(\mathrm{~s})$ should be proportional to the squared anomalous correction for the kth energy, and (ii) the Bragg peaks due to the internal structure should disappear. These conditions were met for all the Ptcontaining samples in Figure 2 except for the samples $\mathrm{Pt} 2$ and $\mathrm{Pt} 3$ reduced in the mother solutions. F or these two samples it was not possible to reliably determine anomalous contributions and to evaluate the volume distribution functions of the nanoparticles. The absence of signal from the metal indicates that either most of the Pt atoms form very large (with the radii exceeding 50-100 nm) conglomerates or they remain in the form of ions or isolated atoms or small clusters. Both very small and very large metal particles or salt clusters would yield no detectable small-angle scattering signal. It is conceivable that the large particles precipitated from the mother solution on the surface of the PODS restrict the accessibility of the siloxy bilayer for the reducing agents, and thus the Pt salts inside the bilayer remain largely nonreduced.

The samples Pt1 (from $\mathrm{K}_{2} \mathrm{PtCl}_{4}$ ) and Pt4 and Pt5 obtained by reduction of $\mathrm{H}_{2} \mathrm{PtCl}_{6}$ after isolation from reaction solutions provide clear and consistent anomalous signals in Figure 3. The size distributions of the nanoparticles computed from these signals are presented in Figure 4. For the sample obtained by the silanol reduction of $\mathrm{K}_{2} \mathrm{PtCl}_{4}$, about $20 \%$ of all Pt atoms form nanoparticles (Table 2). This incomplete reduction can be caused by a shortage of the reducing agent (silanol groups within the siloxy bilayer) although the 
$\mathrm{D}_{\mathrm{v}}(\mathrm{R}), 10^{-5} \mathrm{~cm}^{3} / \mathrm{nm}$

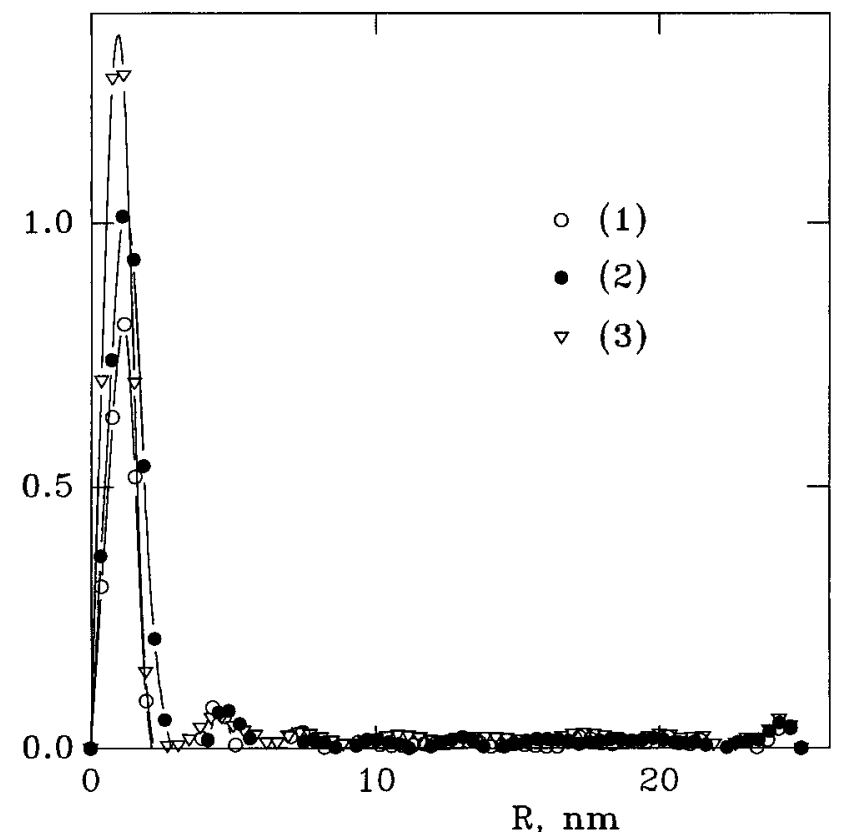

Figure 4. Volume distribution functions of the Pt nanoparticles in PODS. Curves $1-3$ correspond to the samples Pt1, Pt4, and Pt5, respectively.

Table 2. Structural Characteristics of the Initial and Metal-Containing PODS

\begin{tabular}{lcccc}
\hline sample & $\mathrm{S}_{1}, \mathrm{~nm}^{-1}$ & $\mathrm{~L}, \mathrm{~nm}$ & $\Delta / \overline{\mathrm{d}}, \mathrm{nm}$ & $\overline{\mathrm{d}}, \mathrm{nm}$ \\
\hline PODS1 & 1.21 & 37 & 0.120 & $5.24 \pm 0.03$ \\
PODS2 & 1.21 & 37 & 0.120 & $5.24 \pm 0.03$ \\
Pt1 & 1.20 & 37 & 0.120 & $5.25 \pm 0.03$ \\
Pt2 & 1.21 & 36 & 0.120 & $5.23 \pm 0.04$ \\
Pt3 & 1.21 & 35 & 0.121 & $5.22 \pm 0.04$ \\
Pt4 & 1.21 & 38 & 0.117 & $5.22 \pm 0.04$ \\
Pt5 & 1.21 & 36 & 0.120 & $5.22 \pm 0.05$ \\
Au1 & 1.21 & 40 & 0.115 & $5.23 \pm 0.04$ \\
Au2 & 1.21 & 38 & 0.118 & $5.24 \pm 0.04$ \\
Au3 & 1.20 & 36 & 0.120 & $5.25 \pm 0.08$ \\
Au4 & 1.26 & 32 & 0.126 & $4.99 \pm 0.07$ \\
Au5 & 1.21 & 37 & 0.120 & $5.23 \pm 0.06$
\end{tabular}

a $S_{1}$, position of the first maximum; $L$, long-range order dimension; $\Delta / \bar{d}$, degree of disorder; $\bar{d}$, periodicity; PODS1, PODS2, two independent PODS samples.

particles grow up to the limit set by the siloxy bilayer cavity. For the samples obtained after reduction of $\mathrm{PtCl}_{6}{ }^{2-}$ (Pt4 and Pt5), the supply of the both reducing agents is sufficient to provide a higher percentage of the nanoparticle formation (up to $70 \%$ for Pt4). The samples Pt1, Pt4, and Pt5 yiel d nearly identical particle size distributions and display the major fractions (more than $80 \%$ ) of small (radii of about 1-2 nm) particles, which can be accommodated within the siloxy bilayer (see also discussion in the next section). This suggests that formation of nanoparticles does occur inside the siloxy bilayer of PODS where the particle growth is determined by the size of the cavity. This hypothesis is also corroborated by transmission el ectron microscopy (TEM) showing metal particles with diameters of about $2 \mathrm{~nm}$ in regularly spaced darker areas of the micrographs (the TEM results are presented el sewhere ${ }^{29}$ ). The nanoparticle size distributions are largely deter-

(29) Spontak, R.; Bronstein, L.; Wilder, E.; Chernyshov, D.; Valetsky, P. M. Langmuir, submitted. mined by this "cage" effect and are thus independent of the metal compound loading and even of the type of reducing agent. This behavior strongly differs from the one observed by us earlier for other nanostructured systems.5,25,26 In the block copolymer micelles, in both aqueous and nonaqueous media, an increase of the metal compound loading yiel ded larger particles because of an increase of the metal compound concentration in the micelle core. The influence of the reducing agent type was even more pronounced both for micellar systems and for complexes of polyel ectrolyte gels with oppositely charged surfactants: the fast nucleation provided much smaller particles than the slow one. ${ }^{5,14,25,26,28}$ Among all the samples studied earlier, only hyper-cross-linked polystyrene (HPS), containing rigid cavities of about $2 \mathrm{~nm}$ in size, governed the nanoparticle growth independently on metal compound loading and on the rate of the metal particle nucleation. ${ }^{30}$ Similar to the HPS, the crucial parameter for particle growth in the PODS siloxy bilayer seems to be the volume available to the growing particles. Though the siloxy backbones are known to be very flexible, the sheetlike structure formed by the hydrogen bonds and rare cross-links should be able to provide the necessary rigidity of the bilayer to control the size of cavity and nanoparticle growth.

2.1.2. Internal Structure. All samples in Figure 2 display pronounced Bragg peaks due to the ordered internal structure, and Table 2 presents the structural parameters characterizing the ordering. The mean longrange order dimension $\mathrm{L}$ and the degree of disorder $\Delta / \mathrm{d}$ are computed from the first Bragg peak. The former parameter estimates the size of quasi-crystalline zones in the sample whereas the latter provides the relative mean-square deviation of the distance between the neighboring periodic motifs $\Delta / \overline{\mathrm{d}}$. The parameters characterizing the internal ordering of the Pt samples in Table 1 coincide with each other within the experimental error. They are also nearly identical to those computed from the scattering pattern of the initial PODS powder used to prepare the Pt samples (curve $6)$.

As indicated in ref 12, PODS forms a lamellar structure consisting of stacked alkylsiloxane bilayers. The scattering profiles in Figure 2 display up to five peaks at the spacings $d, d / 2, d / 3 \ldots$, and this is fully consistent with a regular lamellar structure. The peak fitting of the SAXS data from the initial PODS powder is illustrated in Figure 5 where the five peaks are shown along with their indices (the fourth-order peak is absent). The periodicity for all the samples in Figure 2 $(5.24 \pm 0.03 \mathrm{~nm})$ is identical within the experimental error.

The electron density profiles within the bilayer were computed from the integral peak intensities using eq 5. The choice of the signs of the reflections was done based on the known chemical composition of PODS. The density profile was expected to steeply increase in the middle of the bilayer due to the silicone atoms. Moreover, a pronounced interval of nearly constant density must be observed in the middle of a monolayer corre sponding to regularly packed alkyl tails. The profiles

(30) Svergun, D. I.; Shtykova, E. V.; Kozin, M. B.; Volkov, V. V.; Dembo, A. T.; Shtykova, E. V., J r.; Bronstein, L. M.; Platonova, O. A.; Yakunin, A. N.; Valetsky, P. M.; Khokhlov, A. R. J . Phys. Chem. B 2000, 104, 5242-5250. 
$\lg I$, relative

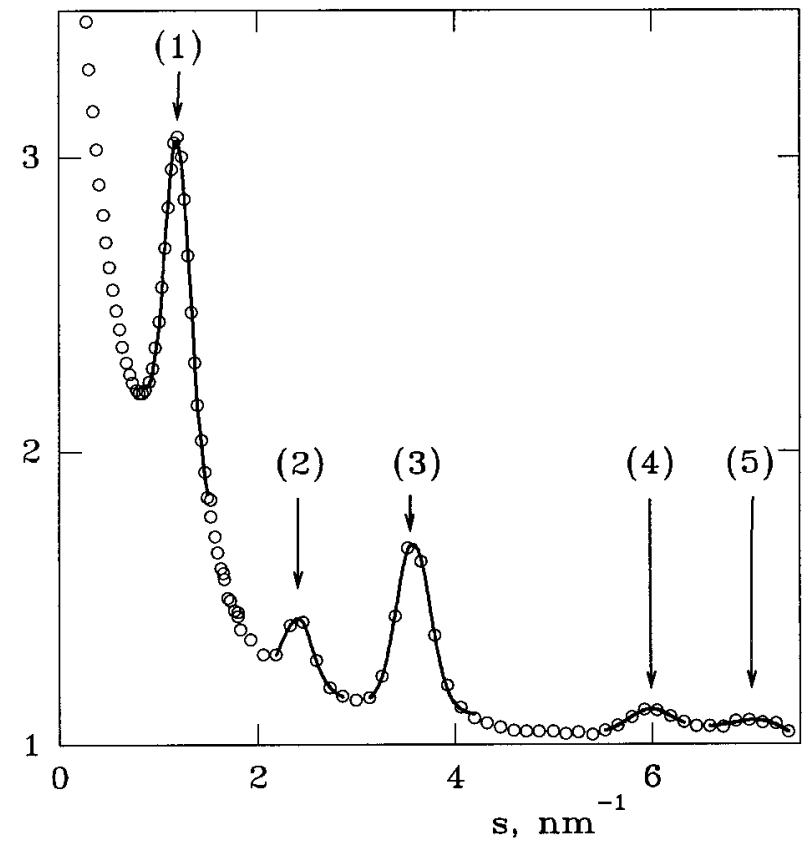

Figure 5. Peak fitting of the scattering pattern from the initial PODS powder. Symbols: experimental curve. Full lines: fitted Gaussian profiles. The peak positions are indicated by arrows, and the orders of reflections are given by numbers.

\section{$\rho$, relative}

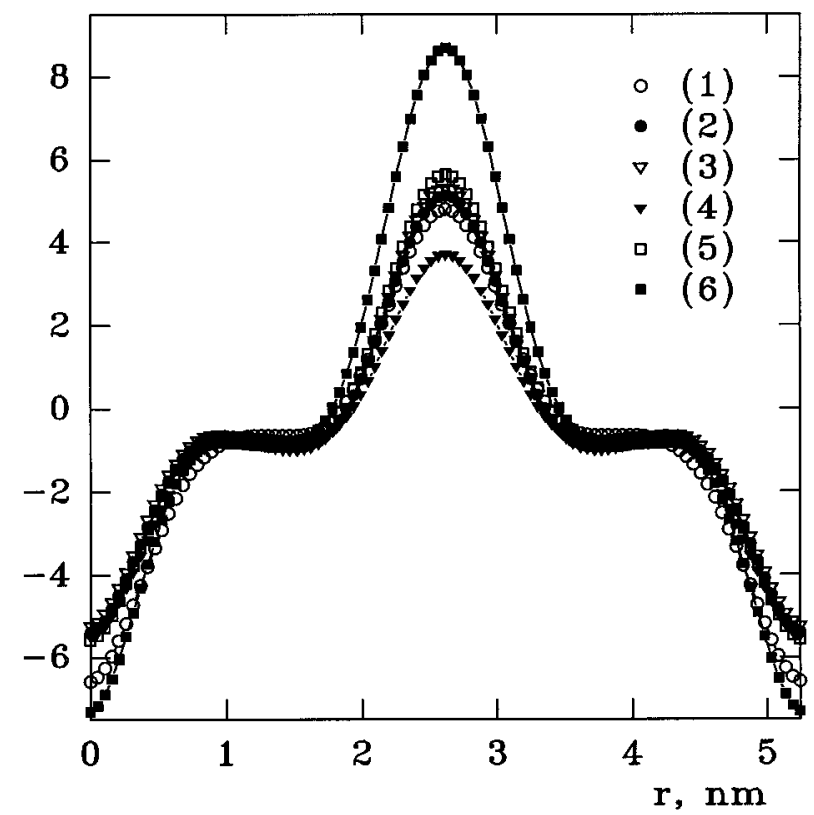

Figure 6. Density profiles perpendicular to the multilayer plane in the Pt-containing PODS. All the profiles are shifted by a constant to best match each other in the range corresponding to the middle of hydrocarbon chains.

presented in Figure 6 were computed with the sign combination -+--- for $\mathrm{I}=1,2,3,5,6$, most consistent with the two requirements. As the value of the zero reflection was not available, this density reconstruction provided the profiles in relative units, and they were appropriately scaled for comparison in Figure 6. Given the low resolution of the profiles, $2 \pi /$ $\mathrm{S}_{\max }=1 \mathrm{~nm}$, the Si atoms in the two monolayers are

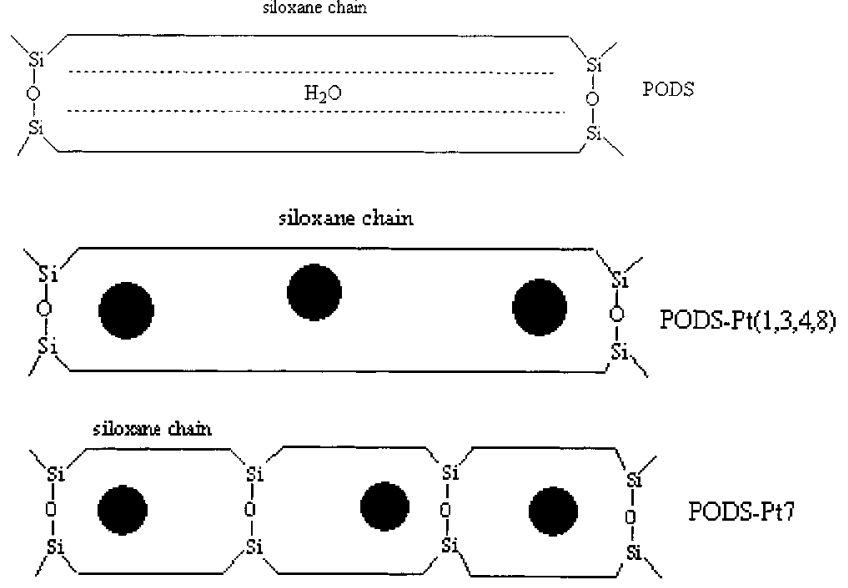

Figure 7. Schematic images of siloxy bilayers for PODS, PODS-Pt1,5, and PODS-Pt4.

not resolved, yielding a single peak in the center of the bilayer. The full width of this peak (about $2 \mathrm{~nm}$ ) correlates well with the sizes of the smaller nanoparticles, suggesting that the siloxy bilayer represents a cage limiting the growth of the nanoparticles. The density outside this peak remains nearly constant up to an offset of about $1.8 \mathrm{~nm}$, reflecting densely packed alkyl tails. Approaching the border of the bilayer, the density decreases, indicating that the tail-tail interface is partially disordered. As seen in Figure 6, the height of the central peak of all the Pt-containing samples is reduced as compared to that for the original PODS. This decrease may be caused by a partial sample disorder due to chemical treatment but also by the presence of nanoparticles in the siloxy bilayer, decreasing the regularity of the repeating density motif. As the largest changes are observed for Pt1, Pt4, and Pt5 samples containing the largest amounts of small nanoparticles, the observed decrease provides strong evidence that the latter do grow within the bilayer. Especially pronounced changes for Pt4 can be explained as follows. The reduction of platinic acid by $\mathrm{H}_{2}$ decreases $\mathrm{pH}$ in the siloxy bilayer due to $\mathrm{HCl}$ formation, which, in turn, should facilitate the silanol group condensation. Then, as schematically illustrated in Figure 7, the siloxy bilayer should contain more cross-links as compared to PODS and to the other PODS-Pt samples.

The periodicity of the PODS lamellae observed by us neatly coincides with the value $5.24 \mathrm{~nm}$ expected for a fully extended bilayer $\mathrm{CH}_{3}\left(\mathrm{CH}_{2}\right)_{18} \mathrm{SiO}-\mathrm{O}_{x} \mathrm{Si}\left(\mathrm{CH}_{2}\right)_{18^{-}}$ $\mathrm{CH}_{3}$. In ref 12 the period of $5.36 \mathrm{~nm}$ was reported, and the $0.12 \mathrm{~nm}$ increase in periodicity was attributed to the presence of a water monolayer trapped between the siloxy groups during the ODS condensation. The authors of ref 12 calculated the periodicity from a simple first peak only without an error estimate, whereas we used the peaks up to sixth order. We believe that our value is more accurate than that reported in ref 12 . The limited resolution of our data does not permit to meaningfully discuss the question of the presence of water inside the siloxy bilayer from the density profiles in Figure 6. Our results do not however contradict a possible presence of the water monolayer. As seen from Figure 6, the ends of the surfactant tails appear to have smaller density; i.e., they are disordered in the bilayer. It is conceivable that such a disorder at the very ends of the octadecyl tails diminishes the period of PODS and thus compensates for the inclusion of the water mono- 
$\lg$ I, relative

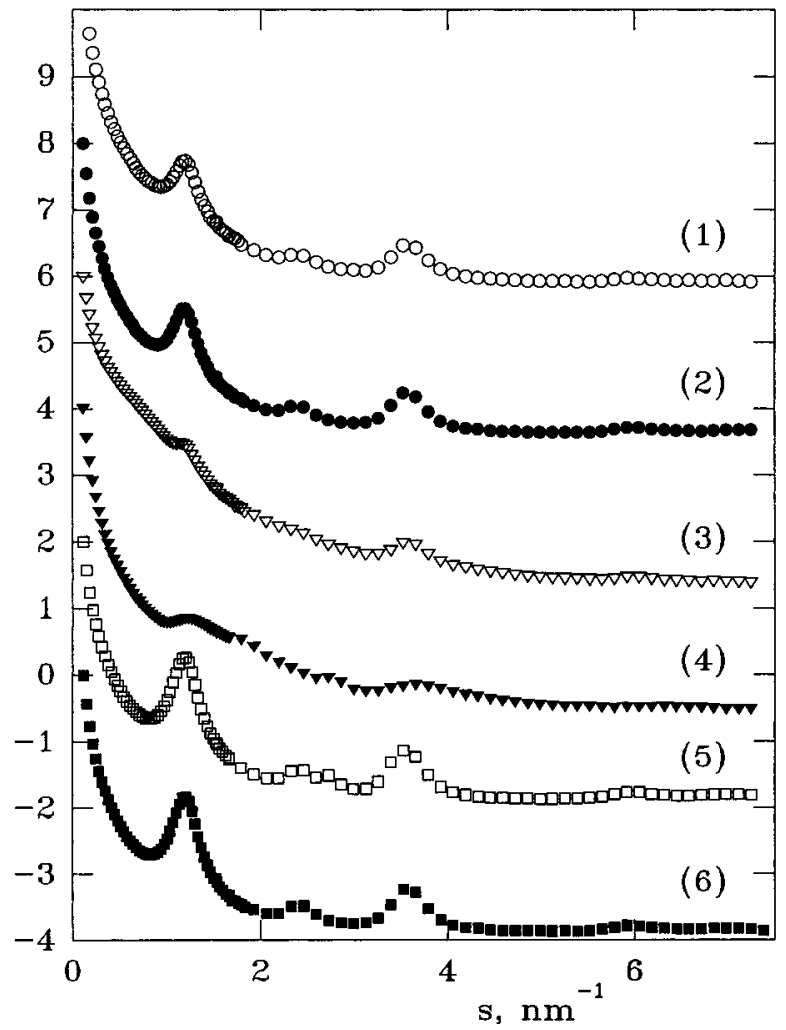

Figure 8. SAXS patterns from the Au-containing PODS samples far from the absorption edge. Curves 1-5 correspond to the samples Au1-Au5, and curve 6 is the scattering from the initial PODS powder. The successive curves are displaced one logarithmic unit down for better visualization. The statistical error bars in the data are smaller than the symbol size.

layer. A similar deviation of the surfactant tail structure from its fully extended length has al ready been reported for lamellar complexes of polyelectrolytes with oppositely charged surfactants. ${ }^{31}$ This deviation could be explained $^{31}$ either by a partial disorder of the alkyl phase structure or by an interpenetration of the surfactant tails.

2.2. Gold-Containing PODS. 2.2.1. SizeDistributions. Figure 8 displays the experimental scattering curves from the Au-containing PODS far from the Au absorption edge. All four Au-containing samples (Au1 to Au4) displayed well-defined anomal ous differences presented in Figure 9, and they were processed to evaluate the volume distribution functions of the nanopartides shown in Figure 10. The sample Au5 (CPC-containing system before metal reduction) taken as a reference does not contain nanoparticles and provides thus no anomal ous signal. The samples Aul and Au2, obtained in mixed solvents where the self-reduction of the gold compound took place within $15 \mathrm{~min}$ after its addition, display bimodal volume distributions where larger (up to $\mathrm{R}=$ $40 \mathrm{~nm}$ ) particles form the major fraction (up to $80 \%$, see Table 2) whereas the contribution of smaller particles is relatively small. This suggests that most of the gold particles grow uncontrolled and are merely deposited on the PODS surface. In contrast, intensive formation of small ( $R<5 \mathrm{~nm}$ ) particles occurs in the PODS/CPC 2633.

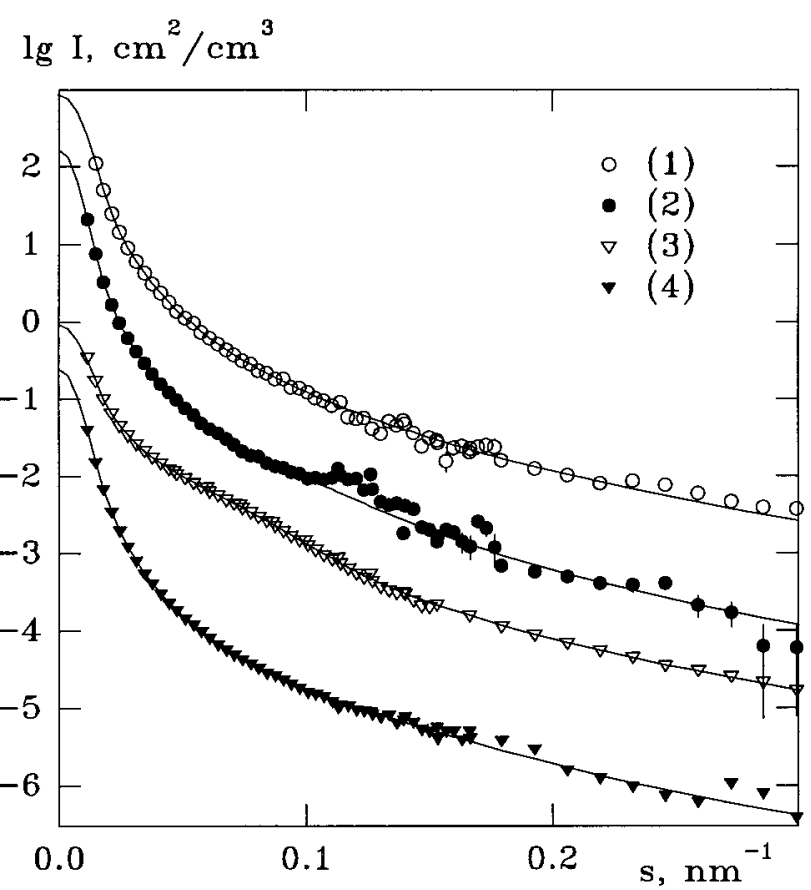

Figure 9. Difference ASAXS data (anomalous signal) from the Au-containing PODS samples. The data sets are numbered as in Figure 8. Symbols with error bars: experimental data. Solid lines: back-transformed curves from the volume distribution functions computed by GNOM. The successive curves are displaced one logarithmic unit down for better visualization.

$D_{\mathrm{v}}(\mathrm{R}), 10^{-5} \mathrm{~cm}^{3} / \mathrm{nm}$

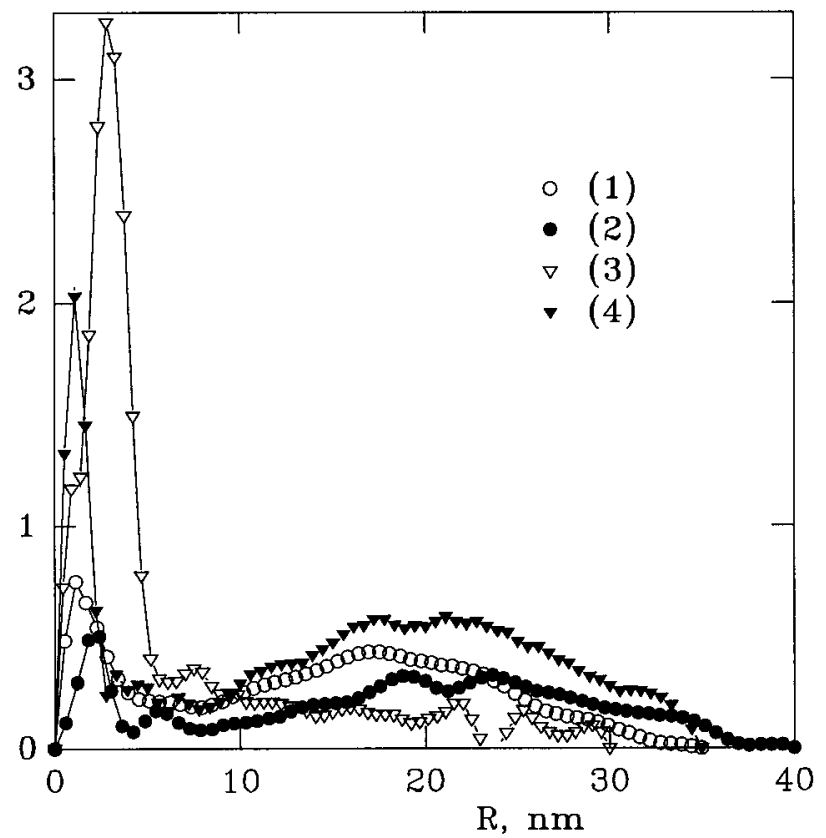

Figure 10. Volume distribution functions of the Au nanoparticles in PODS samples. The numbering of the distributions corresponds to that in Figure 8. The distribution (3) is divided by 3 for clarity.

sample obtained after $\mathrm{NaBH}_{4}$ reduction (Au3). M ost of the particles have radii around $3 \mathrm{~nm}$ whereas larger particles are nearly absent (the volume fraction of small particles is about 70\%). One can also see a fraction of smaller particles with an average radius of $1.5 \mathrm{~nm}$ as a shoulder on the main peak in the volume distribution 


\section{$\rho$, relative}

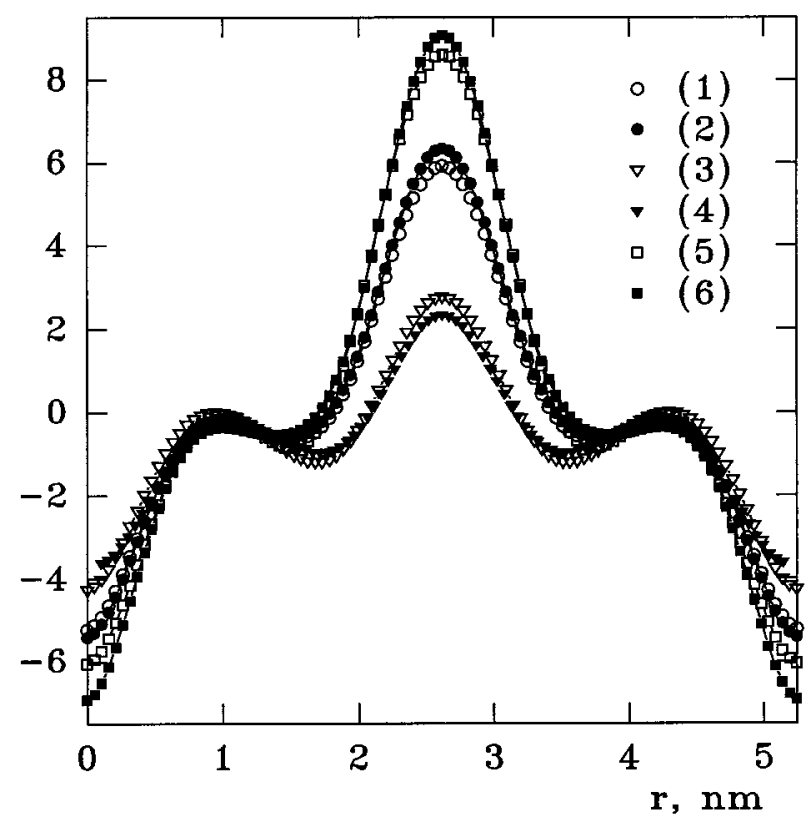

Figure 11. Density profiles perpendicular to the multilayer plane computed by one-dimensional F ourier transformation. The profiles are scaled as in Figure 5.

function (Figure 10, curve 3). This indicates that the major part of particles is formed from the nuclei produced by reduction of $(\mathrm{CP}) \mathrm{AuCl}_{3}(\mathrm{OH})$ (salt of $\mathrm{CPC}$ and gold ion), while a minor portion of nanoparticles with yet smaller sizes is formed in the siloxy bilayer. In the Au4 sample, a significant fraction of small $(\langle\mathrm{R}\rangle$ $=1.5 \mathrm{~nm}$ ) particles is formed, supposedly in the siloxy bilayer, though a broad distribution of larger particles with $\langle R\rangle=20 \mathrm{~nm}$ is also present. Presumably, when the reduction is performed by UV irradiation, slow nucleation allows migration of the nuclei or small clusters located in the hydrophobic layer leading to an uncontrol led aggregation. The blue color of this sample confirms the presence of large gold particles. At the sametime, particles growing in the siloxy bilayer cannot aggregate even at slow nucleation because of the restrictions provided by the bilayer structure.

2.2.2. Internal Structure. Similar to the Pt-containing samples, the scattering patterns in Figure 11 display up to five peaks at spacing $d, d / 2, d / 3 . .$. , and the periodicity of $d=5.24 \pm 0.03 \mathrm{~nm}$ is found for all the structures with the exception of the sample Au4 ( $d=$ $4.99 \mathrm{~nm}$ ). The decrease of the periodicity for the latter sample can be explained by an active silanol group condensation under UV irradiation (see Figure 7) coupled with increase of the sample temperature. This might lead to a sharp increase of the cross-link density, which could expel the water molecules from the siloxy bilayer and thus diminish the period.

The density profiles in the direction perpendicular to the lamella plane are presented in Figure 11. The profiles of the initial PODS and of the nonreduced CPCcontaining sample Au5 (curves 5 and 6) nearly coincide, suggesting that the addition of $\mathrm{CPC}$ and $\mathrm{AuCl}_{3}$ alone, in the absence of nanoparticles, does not affect the bilayer structure. The samples Au3 and Au4 prepared from Au5 via $\mathrm{NaBH}_{4}$ and UV reduction, respectively, exhibit pronounced changes both in siloxy bilayer and in hydrophobic part (curves 3 and 4, respectively). The half-width of the central peak is reduced to about 0.8 $\mathrm{nm}$ for Au3 and to $0.7 \mathrm{~nm}$ for Au4, indicating closer contacts between the Si atoms. The packing of the alkyl chains becomes denser probably because of a significant amount of gold nanoparticles formed in the hydrophobic bilayer. Moreover, for Au4, slow nucleation and growth of particles in the hydrophobic layer are not restricted by the "cage" effect, and the nuclei and small clusters can migrate and aggregate. The nanoparticles can thus grow not only between the alkyl chains but al so between the two hydrophobic layers providing additional distortion of the latter.

\section{Conclusions}

A new hybrid metal/polymer system is developed containing noble metal nanoparticles of well-defined sizes within specific areas of an ordered polymer martix. The use of anomalous small-angle X-ray scattering permitted to comprehensively characterize both the internal structure of the system and the size distributions of the metal nanoparticles. The incorporation of metal compounds in the nanostructured polymer containing siloxy bilayers is strongly determined by the type of metal compound. Planar ions presumably driven by entropy are found to easily penetrate in the thin layers. The entropy gain is provided by the hydrogen bond formation between the chlorine atoms of metal anions and the silanol groups so that each $\mathrm{PtCl}_{4}{ }^{2-}$ ion replaces several water molecules. The sizes of the metal nanoparticles formed in the restricted environment of a siloxy bilayer do not depend on the reducing agent type, on the metal compound loading, and on the metal compound type. This points to a "cage" effect whereby the size of the cavity limits the particle growth. A modification of PODS with CPC allows immobilization of the metal ions both in the siloxy bilayer and in the hydrophobic part of the polymer. In this case, the reduction conditions still do not influence the particles growing in the siloxy bilayer but affect those located in the hydrophobic layers. On the basis of the results, metal-containing PODS with desired properties can be created. Combining hydrophilic and hydrophobic fragments, this hybrid metal-containing polymer can be used for example as heterogeneous catalyst for various long chain substrates with polar groups.

Acknowledgment. The authors acknowledge the financial support provided by the Russian Foundation for Basic Research (Grant 98-03-33372) and by the INTAS (International Association for the Promotion of Cooperation with Scientists from $\mathrm{New}$ Independent States of the former Soviet Union), Grant 96-1115. We thank Dr. G. Goerigk for the assistance in the anomalous scattering measurements, data processing and valuable discussions and R. Kläring for the help with sample holder preparation.

CM001103] 